\title{
Systematic shifts in the variation among host individuals must be considered in climate-disease theory
}

\author{
Joseph R Mihaljevic ${ }^{1 *}$, David J. Páez ${ }^{2}$
}

1. School of Informatics, Computing, and Cyber Systems, Northern Arizona University, Flagstaff, AZ 86011

2. School of Aquatic and Fishery Sciences, The University of Washington, Seattle, 98195

* Corresponding author; e-mail: joseph.mihaljevic@ nau.edu.

Prepared using [ATEX. 


\begin{abstract}
To make more informed predictions of host-pathogen interactions under climate change, studies have incorporated the thermal performance of host, vector, and pathogen traits into disease models. However, this body of work has ignored the fact that disease spread and long-term patterns of host population dynamics are largely determined by the variation in susceptibility among individuals in the host population. Furthermore, and especially for ectothermic host species, variation in susceptibility is likely to be plastic, influenced by variables such as environmental temperature. Quantifying the relationship between temperature and among-host trait variation will therefore be critical for predicting how climate change and disease will interact to influence host-pathogen population dynamics. Here, we demonstrate how short-term effects of temperature on the variation of host susceptibility drive epidemic characteristics, fluctuations in host population sizes, and probabilities of host extinction. We use this quantitative analysis as a basic framework to suggest that more research is needed in disease ecology to understand the mechanisms that shape trait variation, not just trait averages.
\end{abstract}

Manuscript type: Research Article

Keywords: climate, disease, trait variation, epidemiological modeling 


\section{Introduction}

Theory suggests that climate warming can destabilize natural population fluctuations, e.g., moving from stable equilibria or cycles to unstable cycles or chaos [2, 3, 4, 5]. Such destabilization can in turn drive population declines or extinctions, with auxiliary effects that ripple through an ecosystem [6, 7]. Independent of climate, pathogens that cause mortality can also drive periodic fluctuations in population size (e.g., cycling), that can in turn lead to host population decline, local extirpation, or even species extinction [8, 9, 10]. Understanding how climate and host-pathogen interactions combine to determine population declines over the long term is however exceptionally challenging [11, 12, 13]. While there is concern that climate change will exacerbate disease processes such as the frequency, duration and magnitude of epidemics, the mechanisms driving such effects are still poorly characterized, such that our ability to predict climate's future effects on host-pathogen interactions is unclear [14]. To improve our mechanistic understanding, there has been a surge in modeling studies that incorporate the effects of temperature on the plastic traits of hosts and pathogens that drive transmission dynamics. Specifically non-linear thermal performance curves (TPCs, also known as thermal reaction norms) have been incorporated into mechanistic, mathematical models of host-pathogen (or host-vector-pathogen) interactions [15, 16, 17], which has clarified the specific mechanisms by which temperature drives spatial and temporal patterns of pathogen prevalence in a variety of systems [18, 19]. For example, accounting for the temperature-dependence of traits that influence mosquito demography and pathogen transmission has led to well-validated predictions of geographic locations expected to have the highest prevalence of certain mosquito-borne diseases [15, 20, 21, 22, 23]. While much progress has been made to understand the mechanistic effects

of temperature using TPCs and modeling, we argue that the
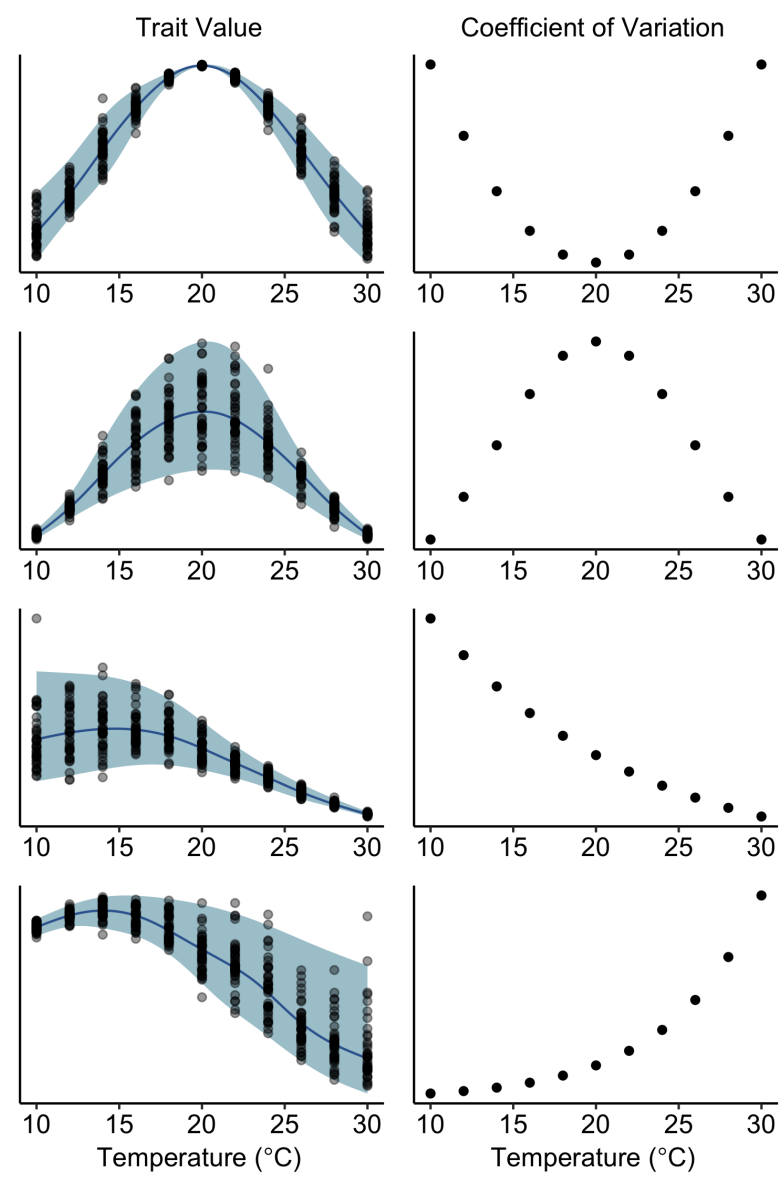

Figure 1: Simulated thermal performance curves in which variability among individuals systematically shifts across the temperature gradient. The left column shows trait values measured across 40 individuals per temperature (points), and the right column shows the coefficient of variation of the trait value among individuals at each temperature. The line is the median (with 95\% confidence ribbon) of an additive quantile regression model fit using the R package qgam [1]. research has largely ignored variation in the shapes of reaction norms among host individuals. As we describe, accounting for how temperature can systematically change the variability in traits expressed among host individuals is likely essential for understanding the ecological outcomes of host-pathogen interactions under climate change.

The variation in trait values among individuals (e.g., transmission rates) has fundamental consequences on disease dynamics [24, 25], yet the vast majority of research on thermal performance curves in disease ecology has focused on population-level trait averages. As we describe here, the variability in plastic trait values among host individuals may also systematically shift along a thermal gradient (Fig. 1), but there is no current theory to understand the implications of temporally shifting trait variation on disease dynamics. In the current literature on temperature-dependent disease ecology, "trait variation" has been used very specifically: the models that integrate TPCs assume that the average values of traits within a population vary as functions of temperature (e.g., solid lines in Fig. 1), and that all individuals share the same trait value at any value of temperature. In other words, "trait variation" is taken to mean that trait averages vary across a temperature gradient. However, because hosts respond 
individualistically to temperature, we must not only be concerned about population-level trait averages, but also with the variation in trait values among individuals. "Trait variation" can therefore also reference the fact that trait values vary among host individuals, as recently emphasized by Cator et al. [18]. However, there has been almost no research on how among-host variability in trait values shifts across temperature gradients. In fact, the among-host variability in trait values may have predictable, functional relationships with temperature that are independent from the relationships between temperature and the average, population-level trait values (Fig. 11). Importantly, we argue that relationships between temperature and among-host trait variation will have meaningful impacts on short- and

long-term disease dynamics that should not be ignored.

Figure 1 illustrates hypothetical examples of how trait variation can change across temperature gradients, independent of trait averages. Across the literature on thermal ecology (even beyond thermal disease ecology), there has been very little focus on quantifying whether temperature affects among-individual variation, but we have found anecdotal data sets that support several of the hypothetical relationships in Figure 1] and others. For example, Careau et al. [27] found that the mean jump time and distance jumped until exhaustion in clawed frogs (Xenopus tropicalis) declined across a temperature gradient, and so did the among-host variation in these traits (see Careau et al. [27]'s Fig. 1c,d, similar to our Fig. 1, third row). Young and Gifford [28] measured the swimming speed of salamanders (Desmognathus brimleyorum) across a temperature gradient, finding a unimodal relationship for the mean, and a higher variation at the peak performance temperature compared to the thermal limits (see Young and Gifford [28]'s Fig. 2, similar to our Fig. 1, second row). The mean maximal sprint speed of common lizards (Zootoca vivipara) increased with temperature, and so did the variation among individuals (see Artacho et al. [29]'s Fig. A1, similar to our Fig. 1. last row, but with an increasing mean).

There are similar anecdotal patterns for vector traits that influence pathogen transmission. Shocket et al. [23] provide several TPCs of mosquito traits, and for some traits, variation among mosquito individuals seems to systematically shift across a thermal gradient For example, the mean lifespan of Culex pipiens declines with temperature and so does variation among host individuals (Fig. 2, top row); the mean infection efficiency of Cx. pipiens for West Nile virus increases with temperature, and the variation seems to be unimodal or generally increases with temperature (Fig. 2, middle row). The original data was not collected in a way to explicitly measure such variation, however, so the patterns are not well resolved for many traits.

In comparison with disease vectors, there are few data sets on the thermal performance of transmission-related traits in ectothermic hosts, making it difficult to generalize how trait variation scales with temperature. Some studies have estimated TPCs for host traits important for immune system dynamics [26, 30, 31, 32]. Yet, while these traits are likely linked to transmission, their specific relationship is unknown. Nevertheless, the data in [26] provide an example of how the variation in traits related to host-pathogen interactions changes with temperature. Overall, 
temperature had a quadratic effect on the likelihood of parasites encysting in the host, and the variation across individuals changed non-linearly as a function of temperature (Fig. 2, bottom row).

100

105

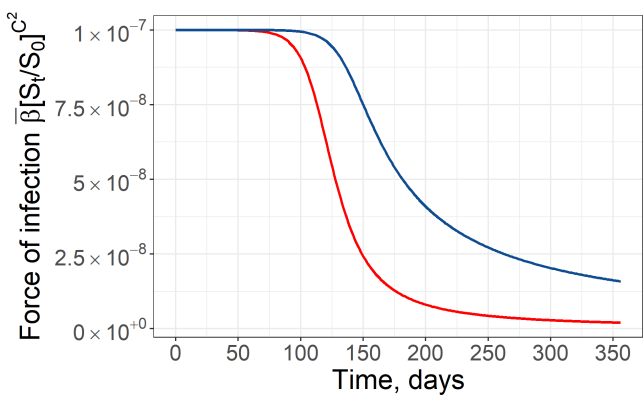

Variation

- High

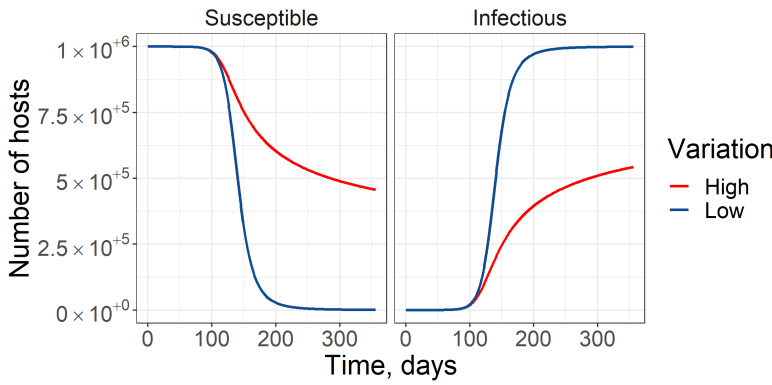

Figure 3: Epizootic dynamics as affected by low and high heterogeneity in susceptibility. The top panel shows how the force of infection changes during an epizootic. The faster decline in the force of infection with high heterogeneity results in fewer hosts contracting infection as shown in the bottom panels. This simulation was conducted using a compartmentalized SusceptibleExposed-Infectious (SEI) model as described in Figure 4. $\bar{\beta}=1 \times 10^{-7}$, blue line $C^{2}=0.25$, red line $C^{2}=5$, $\delta=1 \times 10^{-4}$, Initial population size of susceptible hosts, $S_{0}=1 \times 10^{6}$.

\section{Even if among-host trait variation shifts with temperature, why is it important for our understanding of disease dynamics?}

To address this question, we first describe the role that variation plays in driving host-pathogen population dynamics. We then propose a study framework to investigate temperature-dependent changes in the variation of transmission and to evaluate effects on population dynamics.

Across ecological systems, there is a rising appreciation that the degree to which traits vary among individuals can determine long-term patterns and large-scale processes in ecological time [34, 35, 36, 37, 38]. Indeed, a fundamental result from host-pathogen theory is that, when host individuals vary in traits such as susceptibility or pathogen shedding rate, the magnitude of variation (i.e., high or low variation) determines the size of disease outbreaks and the long-term cycling of disease in host populations [39, 40, 24, 41, 42] (Fig. 3). For example, models that allow for variation in host susceptibility demonstrate that hosts with high resistance and those with high susceptibility have strong effects on epidemic patterns and ultimately how many hosts become infected during an epidemic [25, 43, 44, 45, 46]. At the beginning of outbreaks, the presence of high-susceptibility hosts can lead to faster initial spread of the pathogen, but as the epidemic proceeds, high-resistance hosts generally slow the spread [45]. In fact, high-resistance hosts have disproportionate effects, such that small numbers of these resistant hosts cause epidemics to be smaller, especially in host populations of high density [43, 47, 48]. Moreover, if pathogen infection results in host mortality, even in a moderate fraction of cases, then variation in susceptibility determines how many hosts 
die during an outbreak (Figure 3). Variation in susceptibility therefore has long-term impacts on the cycling of host population abundances and the ultimate threat of pathogens to host population stability and extirpation [43, 49, 45].

This theory that describes how trait variation affects disease dynamics, however, assumes that the values of an individual host's traits are fixed during its lifetime. When host traits related to transmission are plastic and depend on temperature, the assumptions of these previous models are violated and their results are no longer valid. This is problematic because for many host-pathogen systems, epidemics occur while climate is seasonally fluctuating [50, 51]. New theory is therefore needed to evaluate the implications of when variation of traits among host individuals shifts as temperature fluctuates during an epidemic.

\section{Preliminary analysis supports the premise: when trait variation fluctuates with temperature, disease can destabilize host population dynamics and lead to a higher risk of declines.}

We now provide a model analysis to demonstrate how systematic variation in TPCs can drive epidemic dynamics and population cycles. We start with a simple model describing a disease outbreak in a wildlife host with variation in host susceptibility (i.e., some hosts are more resistant and some are more susceptible to infection with the pathogen) [25, 46]. We then expand this simple model to incorporate the effect of varying TPCs on disease outbreaks.

In the simple epizootic model (Figs. 4 and 5), susceptible hosts $(S)$ become infected in a density dependent fashion. Specifically, after a period of incubation, hosts exposed $(E)$ to the pathogen become infectious $(I)$, and infectious hosts shed the pathogen $(P)$ into the environment. Host infection ultimately causes mortality, and pathogens in the environment decay over time.

We then link this epizootic model to a simple model of host reproduction (Fig. 4). We assume that reproduction is discrete in time [43, 52], which is appropriate for host-pathogen systems in which the host has seasonal reproduction. With this structure, the epidemic occurs during the summer ( $\sim 90$ days), after which surviving hosts reproduce and pathogens overwinter. In the next discrete generation, overwintered pathogens infect the susceptible offspring and initiate a new epizootic. Thus, our model assumes that the effect of infection on long-term population dynamics is through host survival.

In this simple model, the crucial process determining the number of surviving hosts is the force of infection, which is described by the distribution of susceptibility in the host population. This distribution is in turn quantified by the average transmission rate, $\bar{\beta}$, and the coefficient of variation in transmission rate among host individuals, $C$. Higher values of $C$ mean that there is higher variation in susceptibility among in175 dividuals. The effect is that individuals with higher susceptibility generally become infected earlier in the epizootic, while the remaining hosts are on average more resistant, such that the force of infection decreases through the epizootic, which can be quantified at time $t$ as $\alpha_{(t)}=\bar{\beta}\left[\frac{S(t)}{S(0)}\right]^{C^{2}}$. This is the mechanism by which high-resistant hosts can slow down an epizootic and ultimately lead to smaller epizootics (Fig. 3).

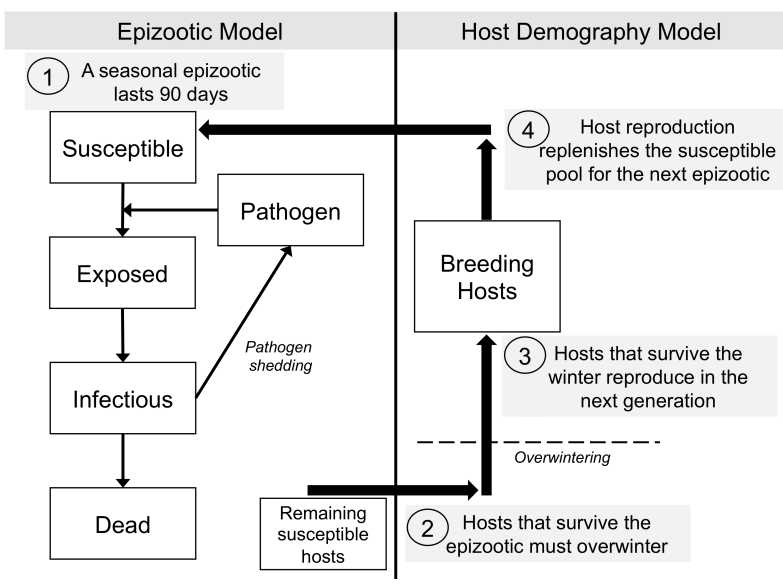

Figure 4: Schematic outlining the linkages between the epizootic and host demography models.

We note that neither the average susceptibility nor the variation in susceptibility are changing across generations because of forces such as selection. The simplified assumptions are thus that environmental conditions exist in each new host generation to generate an equivalent distribution of susceptibilities among host individuals and that the coefficient of variation in transmission rate, $C$, is constant through an epizootic. In addition to determining the number of individuals that become infected and die during an outbreak, the degree of host variation (i.e., the value of $C$ ) determines whether long-term fluctuations in population size follow point equilibrium dynamics or whether they 
cycle in a stable or an unstable manner [25, 43, 45].

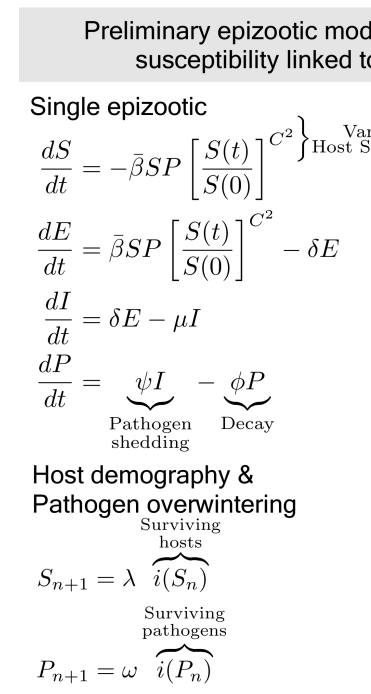

model with variation in
ed to temperature

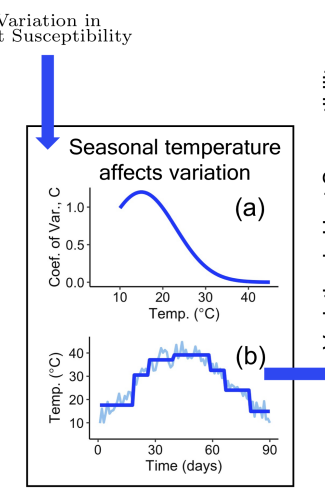

(a)

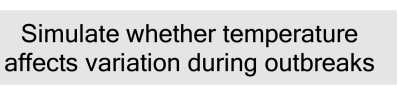

Simulate host-pathogen dynamics across host generations: Host population cycles

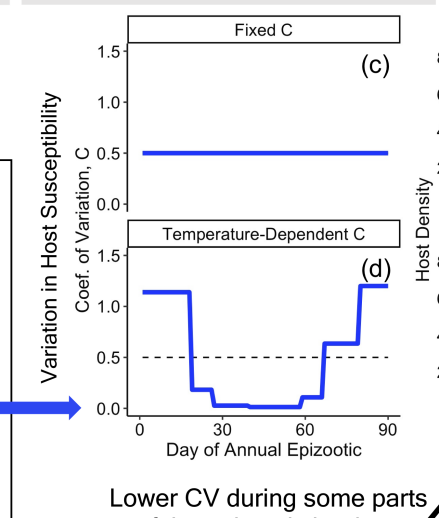
of the epizootic leads to larger outbreaks (fewer high-resistance hosts)

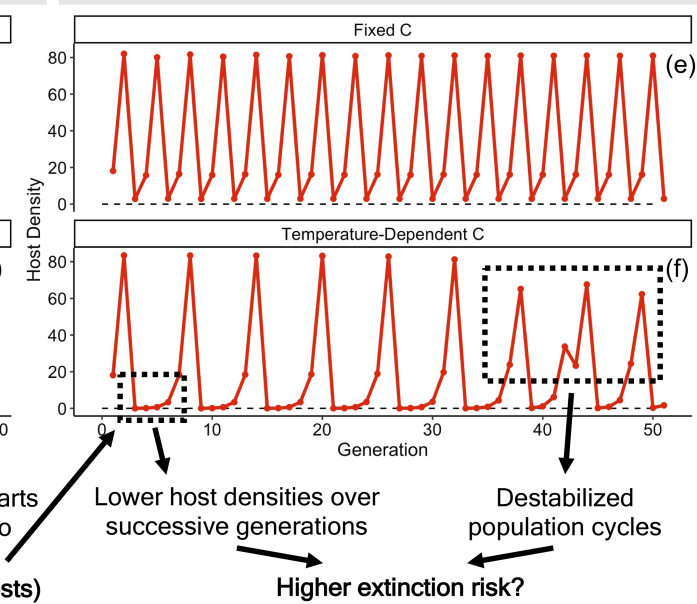

Figure 5: Analyzing a simple model to understand the long-term effects of temperature-dependent trait variation.

Therefore, incorporating the effects of varying TPCs in the epizootic model are expected to result in different epidemic sizes and different long-term patterns of population cycling, even if the effect of temperature on $C$ is transient. To test this hypothesis, we used model simulations to characterize population cycles when the variation in host susceptibility shifts during a seasonal epizootic in response to fluctuating environmental temperature (Fig. 55,b). We, specifically compared two simulations with the same average susceptibility $\bar{\beta}$ but with different effects of temperature on the variation in susceptibility $C$. We either used the simple epizootic model and fixed the variation in susceptibility during the epizootic (Fixed $C, C=0.5$ ), or we allowed this variation to depend on environmental temperature (Temperature-Dependent $C$ ), by assuming that temperature rises and falls across the epizootic period ( 90 days), and by making $C$ a function of this fluctuating temperature $C_{(T)}$. Moreover, we assumed that this heterogeneity function gradually responds to the shifting average temperature over time, rather than shifting daily. To capture this gradual change, we fit a regression tree to simulated temperature data to find the piece-wise average change in temperature, from which we calculated a time-specific value of $C_{(T)}$ (Fig. 5b,d). In this example, we assume that variation $C_{(T)}$ generally declines across a temperature gradient (Fig. $5 \mathrm{k}$ ). To ensure a fair comparison between the two simulations, however, the average $C_{(T)}$ over the epizootic was also 0.5 for the Temperature-Dependent $C$ scenario (horizontal dashed line in (Fig. 5 d).

\section{Modeling results and discussion}

When variation is fixed at 0.5 (Fixed $C$ ), the host population exhibits stable cycles (Fig. 5e), which is expected from previous work [43, 47]. However, when the variation in susceptibility among host individuals declines in hotter parts of the summer (Temperature-Dependent $C$ ), the host abundance cycles are notably different (Fig. 5f). Because temperature reduces variation, the host population temporarily loses some high-resistance individuals; without these individuals, epizootics can become measurably larger on average, especially at high host densities. When the host population is dense, more hosts get infected and die, causing more severe population crashes after peak host densities. There are therefore successive generations of low population numbers, as the host population takes longer to rebound, and the population cycles shift from stable to unstable (Fig. 5f).

Our model analysis suggests that when among-host trait variation shifts systematically across a thermal gradient, we should expect epizootic patterns to deviate from previous theoretical expectations, and, correspondingly, we should expect unique patterns of cycling host abundances. In general, then, our analysis suggests that the relationship between temperature and among-host variation in traits is an important, but overlooked, mechanism by which climate can mediate short- and long-term disease dynamics. Specifically, this finding has several theoretical and 
practical implications.

First, host-pathogen theory shows that when variation in susceptibility (or more generally, infection risk) is environmentally determined and fixed across generations, we expect a stable point equilibrium of host abundance across generations when $C>1$; however, when $C<1$, we expect cycling dynamics (either stable or unstable) [53, 43]. Yet many host populations still cycle when their empirical estimates of $C$ are greater than 1, challenging this foundational theory [47].

In such cases, eco-evolutionary dynamics have been proposed as a mechanism by which variation in susceptibility can be realistically high $(C>1)$ and the host population can still cycle. Specifically, if host susceptibility is heritable, under selection, and is costly to fecundity, then stable cycling can still occur when $C>1$ [47, 49, 45, 42]. Although this eco-evolutionary theory has some empirical support, our analysis introduces an alternative hypothesis: while $C$ may be greater than 1 during parts of the epizootic, if temperature drives $C$ lower than 1 during other parts, we may still expect cycling. More generally, a temperature-dependent $C_{(T)}$ may drive host population cycling in cases it would otherwise be unexpected, although more rigorous theoretical exploration and empirical support of this finding is required.

The short-term effects of temperature on trait variation could also play a major, unappreciated role in determining the threat of pathogens to host population stability in a changing climate. In our analysis, we saw that a temperature-dependent $C_{(T)}$ could result in larger epizootics, leading to successive host generations of low population abundance, as well as destabilized cycling. As the climate becomes warmer and more variable, the relationship between temperature and trait variation could be a strong predictor of how likely a pathogen is to drive a host population to extinction. Temperature-dependent host variation is therefore an unexplored mechanism of host population declines.

\section{Priorities for future research}

Our results underscore the importance of characterizing the distribution of transmission-related traits among host individuals (e.g., in terms of the average susceptibility and the variation in susceptibility), as this will provide fundamental insights into the impact of pathogens on host populations under climate change. Our models are a simplification of the transmission process because temperature is likely to affect the average and the variation in transmission rate, in addition to other parameters that drive epidemic dynamics. We focused on the variation in transmission among host individuals because effects of such variation are mostly omitted from our understanding of disease ecology and of host-pathogen interactions under climate change. Building on the theory presented here is therefore necessary to fully quantify the regulation of disease dynamics by climatic processes.

In general, there has been limited research on the long-term consequences of temperature-dependent traits on host population dynamics in ectotherms. The unpredictability of weather conditions wrought by climate change and the fact that our results show that short-term effects of temperature on host variation can affect long-term population dynamics however suggest that understanding the nature of variation in transmission rates should be a priority of future research. Empirical tests that evaluate the effects of temperatures on transmission rate variability and the variability of other key host and pathogen traits will provide valuable insights into how transmission parameters vary with climate among different taxa. This will prove essential to characterize the potential effects of climate change on host and pathogen populations of concern.

Explicitly measuring effects of temperature on the variation of host traits in a population requires measuring thermal performance curves with adequate replication at each temperature. In addition, studies should ensure that the hosts (or vectors) used in experimentation are a representative sample from the population to capture natural variation. Moreover, it will be important to determine how quickly trait variation among hosts responds to fluctuating temperatures, so that such lags can be adequately incorporated into modeling studies [19]. For some host-pathogen interactions, a close estimate of transmission can be obtained via experimental procedures such as dose-response experiments, where both pathogen dosage and temperature conditions are varied. Models specifically built to estimate transmission parameters can then be applied to these data [25, 54]. However, estimating the effects of temperature on transmission parameters becomes more challenging where experimentation is unfeasible or impractical. In these 
cases, additional methods exist to statistically infer transmission parameters from time-series data collected from lab-based or naturally-occurring epidemics [55, 56, 46]. These studies could also help reveal whether the effects of temperature-dependent trait averages and variances can be detected at large spatial and temporal scales or whether measurements made at smaller scales (e.g., via experimentation) have meaningful effects on large-scale epidemics and long-term host population dynamics in nature.

To further explore the effect of temperature-dependent variation, modeling studies will need to consider how temperature affects the mean and variance of multiple traits simultaneously. And we need to evaluate in which cases temperature-dependent means or variation will be more important for system dynamics. This poses technical and conceptual challenges. First, to capture inter-individual variation, modeling methods must allow individual hosts (and vectors) to have unique responses to temperature, while imposing any known functional relationships between temperature and the averages and variation of specific traits. This can sometimes be done using carefully designed differential equation models (e.g., Cator et al. [18]), however, it may necessitate building more complex, individualbased models. In constructing complex models, it will be important to evaluate model parsimony, so that general insights can still be derived. Further, sensitivity analysis could be used to compare the effect sizes of temperaturedependent means and variation of certain traits to reveal which traits should be of particular interest for specific systems.

Overlooking the effect of systemic changes in the variation of transmission parameters with climatic variables may provide erroneous predictions about the impact of disease on host population dynamics. Accounting for changes in the distribution of transmission parameters (and not only their averages) among host individuals across environmental gradients and time is however possible through experimentation and the implementation of novel statistical techniques. The application of these methods could make direct contributions to understanding risks of extinction of wildlife populations threatened by climate change.

\section{Acknowledgements}

$\mathrm{n} / \mathrm{a}$

\section{Funding}

This work was supported by the State of Arizona Technology and Research Initiative Fund (TRIF), administered by the Arizona Board of Regents, through Northern Arizona University.

\section{Data, code, and materials}

All data and code to run simulations and generate the figures are publicly available on GitHub (https://github. com/joseph-mihaljevic/climate-trait-var)

\section{References}

[1] Matteo Fasiolo, Yannig Goude, Raphael Nedellec, and Simon N. Wood. Fast calibrated additive quantile regression., 2017. URL/https://arxiv.org/abs/1707.03307.

[2] David A. Vasseur and Kevin S. McCann. A mechanistic approach for modeling temperature-dependent consumer-resource dynamics. American Naturalist, 166(2):184-198, 2005. doi: 10.1086/431285.

[3] Jan Ohlberger, Eric Edeline, Leif Asbjørn Vøllestad, Nils C. Stenseth, and David Claessen. Temperature-driven regime shifts in the dynamics of size-structured populations. American Naturalist, 177(2):211-223, 2011. doi: $10.1086 / 657925$.

[4] Mary I. O'Connor, Benjamin Gilbert, and Christopher J. Brown. Theoretical predictions for how temperature affects the dynamics of interacting herbivores and plants. American Naturalist, 178(5):626-638, 2011. doi: $10.1086 / 662171$.

[5] Michela Pacifici, Wendy B. Foden, Piero Visconti, James E.M. Watson, Stuart H.M. Butchart, Kit M. Kovacs, 
Brett R. Scheffers, David G. Hole, Tara G. Martin, H. Resit Akçakaya, Richard T. Corlett, Brian Huntley, David Bickford, Jamie A. Carr, Ary A. Hoffmann, Guy F. Midgley, Paul Pearce-Kelly, Richard G. Pearson, Stephen E. Williams, Stephen G. Willis, Bruce Young, and Carlo Rondinini. Assessing species vulnerability to climate change. Nature Climate Change, 5(3):215-225, 2015. doi: 10.1038/nclimate2448.

[6] Rolf A. Ims, John André Henden, and Siw T. Killengreen. Collapsing population cycles. Trends in Ecology and Evolution, 23(2):79-86, 2008. doi: 10.1016/j.tree.2007.10.010.

[7] Frédéric Barraquand, Stilianos Louca, Karen C. Abbott, Christina A. Cobbold, Flora Cordoleani, Donald L. DeAngelis, Bret D. Elderd, Jeremy W. Fox, Priscilla Greenwood, Frank M. Hilker, Dennis L. Murray, Christopher R. Stieha, Rachel A. Taylor, Kelsey Vitense, Gail S.K. Wolkowicz, and Rebecca C. Tyson. Moving forward in circles: challenges and opportunities in modelling population cycles. Ecology Letters, 20(8):10741092, 2017. doi: 10.1111/ele.12789.

[8] Wesley M Hochachka and André A Dhondt. Density-dependent decline of host abundance resulting from a new infectious disease. Proceedings of the National Academy of Sciences, 97(10):5303-5306, 2000.

[9] Francisco De Castro and Benjamin Bolker. Mechanisms of disease-induced extinction. Ecology Letters, 8(1): 117-126, 2005.

[10] Hamish McCallum. Disease and the dynamics of extinction. Philosophical Transactions of the Royal Society B: Biological Sciences, 367(1604):2828-2839, oct 2012. doi: 10.1098/rstb.2012.0224.

[11] Barry W Brook, Navjot S Sodhi, and Corey JA Bradshaw. Synergies among extinction drivers under global change. Trends in ecology \& evolution, 23(8):453-460, 2008.

[12] KD Lafferty. The ecology of climate change and infectious diseases. Ecology, 90(4):888-900, 2009.

[13] Drew Harvell, Sonia Altizer, Isabella M Cattadori, Laura Harrington, and Ernesto Weil. Climate change and wildlife diseases: when does the host matter the most? Ecology, 90(4):912-920, 2009.

[14] Kevin D. Lafferty and Erin A. Mordecai. The rise and fall of infectious disease in a warmer world. F1000Research, 5:1-8, 2016. doi: 10.12688/F1000RESEARCH.8766.1.

[15] Erin A. Mordecai, Emily de Moor, Kevin D. Lafferty, Samraat Pawar, Christian Balzer, Thomas C. Smith, Leah R. Johnson, Sadie J. Ryan, Krijn P. Paaijmans, Amy McNally, and Tal Ben-Horin. Optimal temperature for malaria transmission is dramatically lower than previously predicted. Ecology Letters, 16(1):22-30, 2012. doi: $10.1111 /$ ele.12015.

[16] Jason R. Rohr, Thomas R. Raffel, Andrew R. Blaustein, Pieter T.J. Johnson, Sara H. Paull, and Suzanne Young. Using physiology to understand climate-driven changes in disease and their implications for conservation. Conservation Physiology, 1(1):1-15, 2013. doi: 10.1093/conphys/cot022.

[17] Péter K. Molnár, Jason P. Sckrabulis, Karie A. Altman, and Thomas R. Raffel. Thermal Performance Curves and the Metabolic Theory of Ecology-A Practical Guide to Models and Experiments for Parasitologists. Journal of Parasitology, 103(5):423, 2017. doi: 10.1645/16-148.

[18] L Cator, Leah R Johnson, Erin A Mordecai, Fadoua El Moustaid, Thomas RC Smallwood, Shannon L LaDeau, Michael A Johansson, Peter J Hudson, Michael Boots, Matthew B Thomas, et al. The role of vector trait variation in vector-borne disease dynamics. Frontiers in Ecology and Evolution, 2020.

[19] Jason R Rohr and Jeremy M Cohen. Understanding how temperature shifts could impact infectious disease. PLoS biology, 18(11):e3000938, 2020. 
[20] Erin A. Mordecai, Jeremy M. Cohen, Michelle V. Evans, Prithvi Gudapati, Leah R. Johnson, Catherine A. Lippi, Kerri Miazgowicz, Courtney C. Murdock, Jason R. Rohr, Sadie J. Ryan, Van Savage, Marta S. Shocket, Anna Stewart Ibarra, Matthew B. Thomas, and Daniel P. Weikel. Detecting the impact of temperature on transmission of Zika, dengue, and chikungunya using mechanistic models. PLoS Neglected Tropical Diseases, 11(4):1-18, 2017. doi: 10.1371/journal.pntd.0005568.

[21] Marta Strecker Shocket, Sadie J. Ryan, and Erin A. Mordecai. Temperature explains broad patterns of Ross River virus transmission. eLife, 7:1-22, 2018. doi: 10.7554/eLife.37762.

[22] Erin A. Mordecai, Jamie M. Caldwell, Marissa K. Grossman, Catherine A. Lippi, Leah R. Johnson, Marco Neira, Jason R. Rohr, Sadie J. Ryan, Van Savage, Marta S. Shocket, Rachel Sippy, Anna M. Stewart Ibarra, Matthew B. Thomas, and Oswaldo Villena. Thermal biology of mosquito-borne disease. Ecology Letters, 22 (10):1690-1708, 2019. doi: 10.1111/ele.13335.

[23] Marta S Shocket, Anna B Verwillow, Mailo G Numazu, Hani Slamani, Jeremy M Cohen, Fadoua El Moustaid, Jason Rohr, Leah R Johnson, and Erin A Mordecai. Transmission of west nile and five other temperate mosquito-borne viruses peaks at temperatures between 23c and 26c. Elife, 9:e58511, 2020.

[24] James O Lloyd-Smith, Sebastian J Schreiber, P Ekkehard Kopp, and Wayne M Getz. Superspreading and the effect of individual variation on disease emergence. Nature, 438(7066):355, 2005.

[25] Greg Dwyer, JS Elkinton, and JP Buonaccorsi. Host Heterogeneity in Susceptibility and Disease Dynamics : Tests of a Mathematical Model. The American Naturalist, 150(6):685-707, 1997.

[26] Karie A Altman, Sara H Paull, Pieter TJ Johnson, Michelle N Golembieski, Jeffrey P Stephens, Bryan E LaFonte, and Thomas R Raffel. Host and parasite thermal acclimation responses depend on the stage of infection. Journal of Animal Ecology, 85(4):1014-1024, 2016.

[27] Vincent Careau, Peter A Biro, Camille Bonneaud, Eric B Fokam, and Anthony Herrel. Individual variation in thermal performance curves: swimming burst speed and jumping endurance in wild-caught tropical clawed frogs. Oecologia, 175(2):471-480, 2014.

[28] Vanessa KH Young and Matthew E Gifford. Limited capacity for acclimation of thermal physiology in a salamander, desmognathus brimleyorum. Journal of Comparative Physiology B, 183(3):409-418, 2013.

[29] Paulina Artacho, Isabelle Jouanneau, and Jean-François Le Galliard. Interindividual variation in thermal sensitivity of maximal sprint speed, thermal behavior, and resting metabolic rate in a lizard. Physiological and Biochemical Zoology, 86(4):458-469, 2013.

[30] Michael W Butler, Zachary R Stahlschmidt, Daniel R Ardia, Scott Davies, Jon Davis, Louis J Guillette Jr, Nicholas Johnson, Stephen D McCormick, Kevin J McGraw, and Dale F DeNardo. Thermal sensitivity of immune function: evidence against a generalist-specialist trade-off among endothermic and ectothermic vertebrates. The American Naturalist, 181(6):761-774, 2013.

[31] Shelley A Adamo and Maggie ME Lovett. Some like it hot: the effects of climate change on reproduction, immune function and disease resistance in the cricket gryllus texensis. Journal of Experimental Biology, 214 (12):1997-2004, 2011.

[32] Frederik Franke, Nadja Raifarth, Joachim Kurtz, and Jörn P Scharsack. Consequences of divergent temperature optima in a host-parasite system. Oikos, 128(6):869-880, 2019.

[33] Bret D. Elderd and James R. Reilly. Warmer temperatures increase disease transmission and outbreak intensity in a host-pathogen system. Journal of Animal Ecology, 83(4):838-849, 2014. doi: 10.1111/1365-2656.12180. 
[34] Daniel I Bolnick, Priyanga Amarasekare, Márcio S Araújo, Reinhard Bürger, Jonathan M Levine, Mark Novak, Volker H W Rudolf, Sebastian J Schreiber, Mark C Urban, and David a Vasseur. Why intraspecific trait variation matters in community ecology. Trends in ecology \& evolution, 26(4), feb 2011. doi: 10.1016/j.tree. 2011.01.009.

[35] W Wesley Dowd, Felicia A King, and Mark W Denny. Thermal variation, thermal extremes and the physiological performance of individuals. Journal of Experimental Biology, 218(12):1956-1967, 2015.

[36] Simone Des Roches, David M Post, Nash E Turley, Joseph K Bailey, Andrew P Hendry, Michael T Kinnison, Jennifer A Schweitzer, and Eric P Palkovacs. The ecological importance of intraspecific variation. Nature ecology \& evolution, 2(1):57-64, 2018.

[37] Suvi Sallinen, Anna Norberg, Hanna Susi, and Anna-Liisa Laine. Intraspecific host variation plays a key role in virus community assembly. Nature communications, 11(1):1-11, 2020.

[38] AC Westerband, JL Funk, and KE Barton. Intraspecific trait variation in plants: a renewed focus on its role in ecological processes. Annals of Botany, 127(4):397-410, 2021.

[39] Sara H. Paull, Sejin Song, Katherine M. McClure, Loren C. Sackett, a. Marm Kilpatrick, and Pieter Tj J Johnson. From superspreaders to disease hotspots: Linking transmission across hosts and space. Frontiers in Ecology and the Environment, 10(2):75-82, mar 2012. doi: 10.1890/110111.

[40] Kimberly L. VanderWaal and Vanessa O. Ezenwa. Heterogeneity in pathogen transmission: mechanisms and methodology. Functional Ecology, 30(10):1606-1622, 2016. doi: 10.1111/1365-2435.12645.

[41] MJ Keeling and Pejman Rohani. Modeling infectious diseases in humans and animals. Princeton University Press, 2008.

[42] Greg Dwyer, JR Mihaljevic, and VM Dukic. Can Eco-Evo Theory Explain Population Cycles in the Field? The American Naturalist, in press.

[43] Greg Dwyer, Jonathan Dushoff, Joseph S Elkinton, and Simon A Levin. Pathogen-driven outbreaks in forest defoliators revisited: building models from experimental data. The American Naturalist, 156(2):105-120, 2000.

[44] Arietta E. Fleming-Davies, Vanja Dukic, Viggo Andreasen, and Greg Dwyer. Effects of host heterogeneity on pathogen diversity and evolution. Ecology Letters, 18(11):1252-1261, 2015. doi: 10.1111/ele.12506.

[45] David Páez and Arietta E. Fleming-Davies. Understanding the Evolutionary Ecology of Host-Pathogen Interactions Provides Insights into the Outcomes of Insect Pest Biocontrol. Viruses, 12(141), 2020. doi: https://doi.org/10.3390/v12020141.

[46] Joseph R. Mihaljevic, Carlos M. Polivka, Constance J. Mehmel, Chentong Li, Vanja Dukic, and Greg Dwyer. An Empirical Test of the Role of Small-Scale Transmission in Large-Scale Disease Dynamics. The American Naturalist, 195(4):616-635, apr 2020. ISSN 0003-0147. doi: 10.1086/707457.

[47] Bret D Elderd, Jonathan Dushoff, and Greg Dwyer. Host-pathogen interactions, insect outbreaks, and natural selection for disease resistance. The American Naturalist, 172(6):829-842, 2008.

[48] Bret D Elderd, Brian J Rehill, Kyle J Haynes, and Greg Dwyer. Induced plant defenses, host-pathogen interactions, and forest insect outbreaks. Proceedings of the National Academy of Sciences, 110(37):14978-14983, 2013. 
[49] David J Páez, Vanja Dukic, Jonathan Dushoff, Arietta Fleming-Davies, and Greg Dwyer. Eco-evolutionary theory and insect outbreaks. The American Naturalist, 189(6):616-629, 2017.

[50] Nicholas C Grassly and Christophe Fraser. Seasonal infectious disease epidemiology. Proceedings of the Royal Society of London B: Biological Sciences, 273(1600):2541-2550, 2006.

[51] Sonia Altizer, Andrew Dobson, Parviez Hosseini, Peter Hudson, Mercedes Pascual, and Pejman Rohani. Seasonality and the dynamics of infectious diseases. Ecology Letters, 9(4):467-484, 2006. doi: 10.1111/j. 1461-0248.2005.00879.x.

[52] Greg Dwyer, Jonathan Dushoff, and Susan Harrell Yee. The combined effects of pathogens and predators on insect outbreaks. Nature, 430(6997):341-345, 2004.

[53] MP Hassell, RM May, SW Pacala, and PL Chesson. The persistence of host-parasitoid associations in patchy environments. i. a general criterion. The American Naturalist, 138(3):568-583, 1991.

[54] Frida Ben-Ami, Roland R Regoes, and Dieter Ebert. A quantitative test of the relationship between parasite dose and infection probability across different host-parasite combinations. Proceedings of the Royal Society B: Biological Sciences, 275(1636):853-859, 2008.

[55] Aaron A King, Dao Nguyen, and Edward L Ionides. Statistical inference for partially observed markov processes via the r package pomp. arXiv preprint arXiv:1509.00503, 2015.

[56] Amanda Minter and Renata Retkute. Approximate bayesian computation for infectious disease modelling. Epidemics, 29:100368, 2019. 